\title{
Telehealth demand trends in COVID-19: an infodemiological evaluation of the top 50 most affected countries
}

\author{
Authors: Mark Wong, ${ }^{A}$ Yih-Chung Tham ${ }^{B}$ and Dinesh Gunasekaran ${ }^{B}$
}

\section{Introduction}

The COVID-19 pandemic has led to urgent calls for the adoption and integration of telehealth solutions into healthcare systems. However, public interest in and demand for telehealth during the pandemic are unknown. We used an infodemiological approach to estimate the demand for telehealth services during COVID-19 globally, focusing on the 50 most affected countries and comparing the demand for such services with the level of information communications technology (ICT) infrastructure available.

\section{Materials and methods}

We used Google Trends, the Baidu Index (China) and the Yandex Keyword Statistics (Russia) to extract data on worldwide and individual countries' telehealth-related internet searches from 1 January to 7 July 2020, and presented them as 'relative search volumes' (RSVs), ranging from 0 (lowest) to 100 (highest). Daily COVID-19 cases and deaths were retrieved from the World Health Organization COVID-19 dashboard (https://covid19.who.int/). ICT development index (IDI) for each country was retrieved to estimate the availability of infrastructure support for telehealth services.

\section{Results and discussion}

As a whole, a spike in worldwide telehealth-related RSVs was observed from 11 March 2020 (peak RSV 76.0), which then tailed off in June-July 2020 (mean RSV 25.8), but remained higher than pre-March RSVs (mean 7.29). Of the 50 most affected countries, $42(84 \%)$ manifested increases in RSVs over the evaluation period, with the highest total RSVs observed within Canada (RSV 100) and the USA (RSV 96). Across all 50 countries, the mean RSV was 18.5 \pm 23.2 (median 9.20; interquartile range (IQR) 5.75-18.68), and the mean IDI value was $5.80 \pm 1.84$ (median 6.05; IQR 4.53-7.23). When evaluating associations between RSV and IDI, the USA and Canada demonstrated both high RSVs and IDIs ( $\geq 7.6$ ). In contrast, European countries had relatively low RSVs (range 3.4-19.5) despite high IDIs (mean of 7.1). Several Latin American (Brazil, Chile, Colombia) and South Asian (India, Bangladesh, Pakistan) countries demonstrated relatively high RSVs (range 13.8-73.3) but low IDIs (mean of 4.0 ), indicating that the demand for telehealth services in these regions may be higher than current levels of infrastructure support.

\section{Conclusion}

There is generally increased interest and demand for telehealth services across the 50 countries most affected by COVID-19. There are also indications that certain countries may not have adequate ICT infrastructure to cope with this demand surge for telehealth services. These findings highlight the need to scale up telehealth capabilities, during and beyond COVID-19.

\section{Conflicts of interest}

None declared.
Authors: ${ }^{A}$ Cambridge University, Cambridge, UK; ${ }^{B}$ Singapore Eye Research Institute, Singapore 